\title{
User Configuration of Activity Awareness
}

\author{
Tony McBryan ${ }^{1}$, Philip Gray ${ }^{1}$ \\ ${ }^{1}$ Department of Computing Science, \\ University of Glasgow, Lilybank Gardens, Glasgow, G12 8QQ, UK \\ \{mcbryan,pdg\}@dcs.gla.ac.uk
}

\begin{abstract}
This paper describes an approach to user configuration of activity awareness. This approach offers users increased flexibility by allowing the use of multiple methods of configuration to be used within a unified framework; these configuration methods can include context, policy rules and automatic techniques of configuring behaviour. In this paper we describe the benefits of this flexibility and discuss a model of configuration that can be used to support these features.
\end{abstract}

Keywords: user configuration, activity awareness, configuration, ubiquitous systems

\section{Introduction}

The goal of activity awareness is to allow a user to share their information about their current status or recent activities with other people. Activity Awareness is crucial in CSCW (Computer Supported Collaborative Work) [1] but is also increasingly being used within social contexts and a multitude of online services are available to support this interaction such as Facebook ${ }^{1}$ and Twitter $^{2}$.

Within an assisted living environment, activity awareness allows people to keep informed about the status of a friend, relative or spouse. Knowledge that their loved one has been recently active can relieve stress or worry about their status and can also serve as an early warning indicator

With the typical social networking applications a user can select to add a "friend" and that friend is then able to receive any typed messages that the user enters into the application. However, within a typical assisted living environment there is often the wish to discriminate between which friends receive what type of information as well as what level of detail they may receive. Furthermore, activity information can come from numerous sources within the home; in addition to messages that the user can enter themselves it could also be possible to track the users location or movement, monitor if they have taken medication on schedule, or if they have left their home. Determining which group of people is eligible to receive which activity messages is not a trivial task.

\footnotetext{
${ }^{1} \mathrm{http}: / / \mathrm{www}$. facebook.com

${ }^{2}$ http://www.twitter.com
} 
In addition to this we can recall that social networking is a bidirectional communication channel; it is not only the person living in the home that is likely to generate messages. A grandparent may wish to know that their children and grandchildren are well. They will likely have multiple sources of messages from other people and they may attach different priorities to these messages; or wish to have them delivered in a variety of different ways. Activities from a distant friend may simply be added to a GUI presenting a list of received messages whilst a new message from a close relative may elicit more immediate attention through the use of speech or audio notifications.

However, these additional requirements make the configuration of an activity awareness application much more complicated as the user now needs to specify the precise relationship between activity message inputs (which may be automatic sensors, user controlled or sourced from other people) and outputs (which may be a range of devices or other people we wish to receive the message).

This paper presents an application that is designed to explore these issues.

\section{Related Work}

The need for support of social connections between elder parents and their children is identified by Kidd et al.[2] and identifies the need for understanding how people interact with technology. We argue that configuration is a clear instance of coexistence with technology that requires investigation.

There are a number of existing configuration techniques that have been used for configuration of general purpose ubiquitous systems. These include; Automatic metric based on empirical studies (i.e. Quantitative results such as error rate), Utility metric based on combinations of multiple metrics[3], Context based rules[4], Policy based rules[5], User preferences (expressed a priori), User interaction (expressed at runtime) and Recommenders/Pattern recognition systems[6]. Each technique has various advantages and disadvantages which can make them more or less suited to particular situations; for example utility based metrics may provide overall "good" results in most situations and are easy to configure but may not be suitable in edge case situations.

\section{The Application}

The core idea of this application is to integrate the selection of different configuration techniques current used in ubiquitous systems and to make them available within a unified framework such that a user can choose the technique most appropriate for dealing with a particular aspect of the configuration.

To do this we treat configuration not as a set of static values which the user may set but instead as a function which is designed to return the values to use in any given circumstance. 
We have previously developed an approach to supporting configuration in general ubiquitous systems[7] where we introduce the concept of evaluation functions which can be used to unify the techniques previously discussed.

The details of this technique are outside the scope of this paper but briefly an evaluation function is a function which accepts as parameters a set of configuration possibilities which are determined automatically by the system based on the available devices and friend relationships. The purpose of an evaluation function is to select which of the available configuration possibilities should be used to deliver or receive information. By pairing two evaluation functions together (one for input and one for output) we have a flexible method for allowing the user to specify where to receive activity messages from, and when activities are received to specify what should be done with them.

To investigate this we have developed a Java based application. This application allows sensing from a number of sensors designed to record user activity ambiently such as wireless accelerometers (known as the JAKE and SHAKE devices), webcam movement and monitoring the amount of time since the keyboard or mouse were last used. Additionally these activity messages could be rendered locally through interaction outputs such as a GUI display, a speech synthesis application, through auditory icons or sent to remote outputs such as an email account. Additionally, activity messages could be delivered to other users of the application who could decide how to render these messages using the same configuration methods.

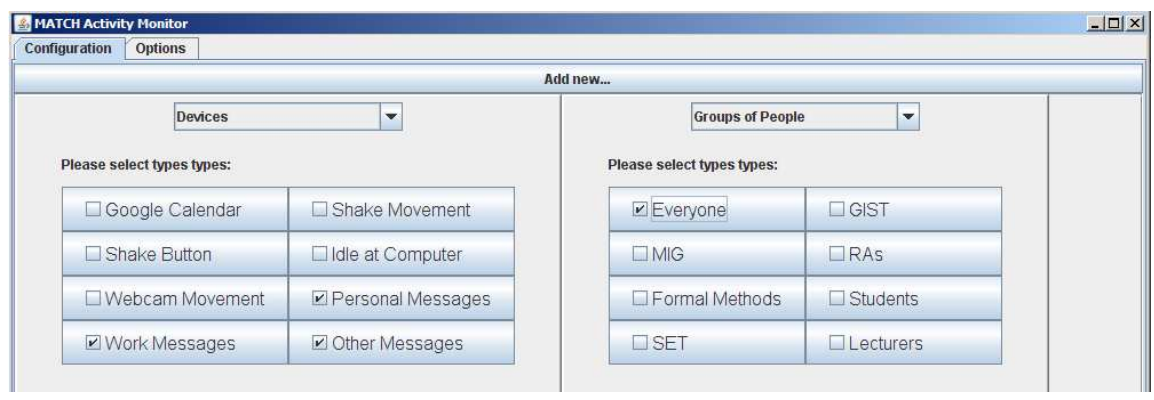

Fig. 1. A sample screen shot of one possible rule where user typed messages classified as "Personal", "Work" and "Other" are delivered to all friends using the "Devices" evaluation function and the "Groups of People" evaluation function. The Devices evaluation function also allows for selection of automatic Calendar events and detected Movement among other options while the Groups of People function allows different groupings of people to be selected.

Users of the application have the capability to select input sources and output destinations in a variety of different ways. Briefly these are selecting by Devices specifically, selecting Groups of Devices, selecting Individual People as well as Preset Groups of People and User Editable Groups of People. Three recommendation systems are included; the first of which automatically selects appropriate possibilities based on the users local usage history, the second selects appropriate possibilities based on a collaborative history of usage across all participants and the third of which simply displays the set of possibilities for the user to select from but also indicates 
their popularity in the current situation based on the collaborative usage history. Furthermore, the user can combine multiple evaluation functions within a single rule (using the combine function which takes two other functions as parameters) and can also create context sensitive rules based on the time since the users last keystroke. In the case of the context sensitive function this also takes two other functions as parameters and executes one or the other based on the situation.

\section{Summary}

In this paper we have presented an application that is currently work in progress. This application is currently being evaluated in qualitative investigations to determine the criteria that a user deems important during the configuration task. The specific questions we have set out to answer are; (i) which configuration techniques are preferred and why?, (ii) what process do users undertake when configuring complex systems?, (iii) what relationship exists between privacy, control and flexibility and which of these do users favour? and (iv) how do users identify and correct unsuitable configurations?

Acknowledgments. This research was carried out within the MATCH (Mobilising Advanced Technologies for Care at Home) Project funded by Scottish Funding Council (grant HR04016). We wish to thank our MATCH colleagues for their contribution to the ideas presented here and for their work in developing the MATCH software framework.

\section{References}

1. Dourish, P. and Bellotti, V. Awareness and Coordination in Shared Workspaces, pp. 107114, Proceedings of the Conference on Computer-Supported Cooperative Work, ACM, Toronto, Canada, November 1992, 0897915429.

2. Kidd, C.D., Orr, R., Abowd, G.D., Atkeson, C.G., Essa, I.A., MacIntyre, B., Mynatt,E., Starner, T.E., and Newstetter, W. The Aware Home: A Living Laboratory for Ubiquitous Computing Research, LNCS, Volume 1670/1999, ISBN 978-3-540-66596-0, pg 191-198, 1999.

3. Sousa, J.P. and Garlan, D.: Improving User-Awareness by Factoring it Out of Applications. Proc System Support for Ubiquitous Computing Workshop (UbiSys), (2003)

4. Calvary, G., Coutaz, J., Daassi, O., Balme, L. and Demeure, A.: Towards a new generation of widgets for supporting software plasticity: the "comet". Preproceedings of EHCI/DSVIS, 4:41--60, (2004)

5. Sohn, T., Dey, A. K.: iCAP: An Informal Tool for Interactive Prototyping of Context-Aware Applications. In: Extended Abstracts of ACM Conference on Human Factors in Computing Systems (CHI 2003). ACM Press, New York (2003) 974-975

6. Bell, M., Hall, M., Chalmers, M., Gray, P. and Brown, B.: Domino: Exploring Mobile Collaborative Software Adaptation. LNCS, (2006)

7. McBryan, T. and Gray, P. A Model-Based Approach to Supporting Configuration in Ubiquitous Systems. In Design, Specification and Verification of Interactive Systems 2008, Ontario, Canada, 2008. 